Pacific Journal of Mathematics

WEAK CONTAINMENT AND KRONECKER PRODUCTS O 


\title{
WEAK CONTAINMENT AND KRONECKER PRODUCTS OF GROUP REPRESENTATIONS
}

\author{
J. M. G. FeLL
}

Introduction. Throughout this paper $G$ is a fixed locally compact group. Let us recall some concepts bearing on the representation theory of $G$. The family of all unitary equivalence classes of unitary representations of $G$ will be called $\mathscr{T}(G)$. A function $\varphi$ of positive type on $G$ is associated with a subset $\mathscr{S}$ of $\mathscr{T}(G)$ if there is an $S$ in $\mathscr{S}$, and a vector $\xi$ in the space $H(S)$ of $S$, such that $\varphi(x)=\left(S_{x} \xi, \xi\right)$ for all $x$ in $G$. An element $T$ of $\mathscr{T}(G)$ is weakly contained in a subset $\mathscr{S}$ of $\mathscr{T}(G)$ if every function of positive type on $G$ associated with $T$ can be approximated uniformly on compact sets by sums of functions of positive type associated with $\mathscr{S}$. The notion of weak containment leads to that of the inner hull-kernel topology of $\mathscr{T}(G)$ : A net $\left\{T^{i}\right\}$ of elements of $\mathscr{T}(G)$ converges to $T$ in this topology if and only if every subnet of $\left\{T^{i}\right\}$ weakly contains $T$. Relativized to the subset $\hat{G}$ of $\mathscr{T}(G)$ consisting of the irreducible representations of $G$, this topology becomes the ordinary hull-kernel topology of $\hat{G}$. (For these notions and facts see [1] and [2]).

If $H$ is a Hilbert space, the adjoint space $\bar{H}$ of $H$ can be defined as the Hilbert space whose underlying set is the same as that of $H$, and which is conjugate-isomorphic with $H$ under the identity map. If $T$ is a unitary representation of $G$, the adjoint representation $\bar{T}$ is defined by the requirements: $H(\bar{T})=H(T)^{-}, \bar{T}_{x}=T_{x}(x \in G)$. The Kronecker product $S \otimes T$ of two unitary representations $S$ and $T$ of $G$ is that representation whose space is $H(S) \otimes H(T)$, and for which $(S \otimes T)_{x}(\xi \otimes \eta)=\left(S_{x} \xi\right) \otimes\left(T_{x} \eta\right)$. We can also describe the Kronecker product $S \otimes \bar{T}$ as follows: $H(S \otimes \bar{T})$ is the Hilbert space of all HilbertSchmidt operators on $H(T)$ to $H(S)$, and $(S \otimes \bar{T})_{x}(A)=S_{x} A T_{x}^{-1}$.

If $\mathscr{S} \subset \mathscr{T}(G)$ and $\mathscr{T} \subset \mathscr{T}(G)$, let $\mathscr{S} \otimes \mathscr{T}$ denote $\{S \otimes T \mid S \in \mathscr{S}$, $T \in \mathscr{T}\}$.

Throughout this paper $I$ will be the one-dimensional identity representation of $G$. It is well known and easily verified that if $S$ and $T$ are finite-dimensional unitary representations of $G$ and $T$ is irreducible, $S \otimes \bar{T}$ contains $I$ if and only if $S$ contains $T$. Can this be generalized to the case where $S$ and $T$ are infinite-dimensional and 'containment' is replaced by 'weak containment'? The main object of this note is to answer this question affirmatively for the case that $S$ is infinitedimensional but $T$ is still finite-dimensional (Theorem 4). In preparation for this we shall show (Theorem 2) that the Kronecker product oper-

Received January 21, 1963. 
ation is continuous with respect to the inner hull-kernel topology of $\mathscr{T}(G)$.

Another by-product of the main result is the following strenthening (Theorem 3) of a remark of Godement ([4], p. 77): If the regular representation $R$ of $G$ weakly contains some finite-dimensional irreducible unitary representation of $G$, then $R$ weakly contains all unitary representations of $G$.

\section{The continuity of the Kronecker product.}

Lemma 1. Suppose that $\mathscr{S} \subset \mathscr{T}(G)$ and $T \in \mathscr{T}(G)$; and let $K$ be the set of all those $\xi$ in $H(T)$ such that the function $\varphi$ defined by $\varphi(x)=\left(T_{x} \xi, \xi\right)(x \in G)$ can be approximated, uniformly on compact sets, by sums of functions of positive type associated with $\mathscr{S}$. Then $K$ is a closed T-invariant linear subspace of $H(T)$.

Proof. Obviously $K$ is closed in the norm and under scalar multiplication. By the easy argument of [1], p. 368, (ii'), $\sum_{i=1}^{n} a_{i} T_{x_{i}} \xi$ is in $K$ whenever $\xi \in K$, the $x_{i}$ are in $G$, and the $a_{i}$ are complex; in particular $K$ is $T$-invariant. It remains only to show $K$ closed under addition.

Let $\xi$ and $\eta$ be elements of $K$; let $L$ and $M$ be the closed invariant subspaces of $H(T)$ generated by $\xi$ and $\eta$ respectively; and let $Q$ be the closure of $L+M$. By the preceding paragraph

$$
L \subset K \text { and } M \subset K \text {. }
$$

If $A$ is projection onto $L^{\perp}, A(M)$ is a dense subspace of $Q \cap L^{\perp}$. So by Mackey's form of Schur's Lemma ([7], Theorem 1.2), the restriction of $T$ to the invariant subspace $Q \cap L^{\perp}$ is equivalent to a subrepresentation of the restriction of $T$ to $M$. This and (1) show that

$$
Q \cap L^{\perp} \subset K \text {. }
$$

Putting $\zeta=\xi+\eta$, we have $\zeta=\xi^{\prime}+\eta^{\prime}$, where $\xi^{\prime} \in L$ and $\eta^{\prime} \in Q \cap L^{\perp}$. Since $L$ and $Q \cap L^{\perp}$ are orthogonal and T-invariant,

$$
\left(T_{x} \zeta, \zeta\right)=\left(T_{x} \xi^{\prime}, \xi^{\prime}\right)+\left(T_{x} \eta^{\prime}, \eta^{\prime}\right)
$$

$(x \in G)$. By (1) and (2) $\xi^{\prime}$ and $\eta^{\prime}$ are in $K$; so by (3) $\zeta \in K$, and $K$ is closed under addition.

REMARK 1. If $A$ is a $C^{*}$-algebra, $\mathscr{T}(A)$ is defined as the set of all equivalence classes of ${ }^{*}$-representations of $A$. Exactly the same proof shows that Lemma 1 is valid for $C^{*}$-algebras, provided that we replace functions of positive type by positive functionals, and uniform approximation on compact sets by weak* approximation. 
REMARK 2. According to Lemma $1, T$ will be weakly contained in $\mathscr{S}$ provided $H(T)$ is generated (under $T$ ) by those $\xi$ in $H(T)$ whose associated functions of positive type are approximated by sums of functions of positive type associated with $\mathscr{S}$. For example, we have immediately:

THEOREM 1. Suppose that $\mathscr{S}_{k} \subset \mathscr{T}(G)$ and $\mathscr{S}_{k}$ weakly contains $T_{k}(k=1,2)$. Then $\mathscr{S}_{1} \otimes \mathscr{S}_{2}$ weakly contains $T_{1} \otimes T_{2}$.

THEOREM 2. The map $\langle S, T\rangle \rightarrow S \otimes T$ (of $\mathscr{T}(G) \times \mathscr{T}(G)$ into $\mathscr{T}(G))$ is continuous with respect to the inner hull-kernel topology of $\mathscr{T}(G)$.

Proof. Let $S^{i} \rightarrow S$ and $T^{i} \rightarrow T$ in $\mathscr{T}(G)$. By the definition of the topology of $\mathscr{T}(G)$, we have only to show that the net $\left\{S^{i} \otimes T^{i}\right\}$ (and hence by the same argument every subnet of it) weakly contains $S \otimes T$. But Theorem 2.2 of [2] clearly shows that the function of positive type associated with each product vector $\xi \otimes \eta$ in $H(S) \otimes H(T)$ can be approximated by functions of positive type associated with the $S^{i} \otimes T^{i}$. Hence by Lemma $1 S \otimes T$ is weakly contained in $\left\{S^{i} \otimes T^{i}\right\}$.

It should be mentioned that the "easy verification" of the proposition used in the proof of [2], p. 260, Corollary 1, actually requires the above Theorem 1 .

2. When does $S \otimes \bar{T}$ weakly contain $I$ ? In this section $G$ is assumed to satisfy the second axiom of countability; and we shall consider only unitary representations acting in a separable space.

Suppose that $T \in \hat{G}$ and $S \in \mathscr{T}(G)$. Is it true that $S \otimes \bar{T}$ weakly contains $I$ if and only if $S$ weakly contains $T$ ? In general, as we next show, the implication is false in both directions, even if $S$ is assumed irreducible.

Let $R$ be the regular representation of $G$, and $T$ some irreducible representation weakly contained in $R$. Clearly $R \cong \bar{R}$. By [6], Theorem 12.2, $R \otimes R$ is a multiple of $R$. So $R \otimes \bar{R}$ weakly contains $I$ if and only if $R$ does. Choose $G$ so that $R$ does not weakly contain $I$; for example $G$ might be the free group on two generators, or a non-compact connected semisimple Lie group (see [8]). Then $R \otimes \bar{R}$ does not weakly contain $I$, and hence, by Theorem 1 , nor does $T \otimes \bar{T}$.

For an easy counter-example in the other direction take $G$ to be the " $a x+b$ " group, and $T$ to be one of the two infinite-dimensional irreducible representations of $G$. Then $\bar{T}=I \otimes \bar{T}$ weakly contains $I$ (see [2], Theorem 5.1), but $I$ does not weakly contain $T$. A "better" example, in which $S \otimes \bar{T}$ weakly contains $I$ but neither $S$ nor $T$ weakly contains the other, will be given in $\S 3$. 
However, if $T$ is finite-dimensional, the answer to the question posed above is affirmative (Theorem 4).

LEMma 2. If $\mathscr{S} \subset \mathscr{T}(G)$ and $\mathscr{S}$ weakly contains a finite-dimensional irreducible unitary representation $T$ of $G$, then $\mathscr{S} \otimes \bar{T}$ weakly contains $I$.

Proof. $\mathscr{S} \otimes \bar{T}$ weakly contains $T \otimes \bar{T}$ by Theorem 1. Since $T$. is finite-dimensional, $T \otimes \bar{T}$ contains $I$.

Here is an interesting consequence of Lemma 2 :

THEOREM 3. If the regular representation $R$ of $G$ weakly contains some finite-dimensional irreducible representation $T$ of $G$, it weakly contains all unitary representations of $G$.

Proof. By Lemma $2 R \otimes \bar{T}$ weakly contains $I$. But by [2], Lemma 4.2, $R \otimes \bar{T}$ is a multiple of $R$. Hence $R$ weakly contains $I$, and the conclusion follows from Godement's remark ([4], p. 77, or [2], p. 260).

Lemma 3. Let $T$ be an irreducible finite-dimensional unitary representation of $G$. To each $\delta>0$, there is a finite subset $F$ of $G$ and an $\varepsilon>0$ such that, whenever $A$ is a positive linear operator on $H(T)$ satisfying (i) $\|A\|=1$ and (ii) $\left\|A T_{x}-T_{x} A\right\|<\varepsilon$ for all $x$ in $F$, then $\|A-E\|<\delta$ ( $E$ being the identity operator on $H(T)$ ).

Proof. Assume the lemma false. Then there is a $\delta>0$ and a net $\left\{A_{i}\right\}$ of positive operators in $Q$ such that $A_{i} T_{x}-T_{x} A_{i} \underset{i}{\longrightarrow} 0$ for all $x$ in $G$; here $Q$ is the compact set of those positive operators $A$ on $H(T)$ for which $\|A\|=1$ and $\|A-E\| \geqq \delta$. Replacing $\left\{A_{i}\right\}$ by a subnet, we may assume that $A_{i} \rightarrow A$ in $Q$. Passing to the limit, we deduce that $A T_{x}=T_{x} A$ for all $x$, whence $A=\lambda E$. Since $A$ is positive and of norm 1 , we must have $\lambda=1$; but this contradicts $\|A-E\| \geqq \delta$.

Lemma 4. Suppose that $\mathscr{S} \subset \mathscr{T}(G)$, and $T$ is a finite-dimensional irreducible unitary representation of $G$ such that $\mathscr{S} \otimes \bar{T}$ weakly contains $I$. Then $\mathscr{S}$ weakly contains $T$.

Proof. The family of all finite direct sums of elements of $\mathscr{S}$ weakly contains $T$ if and only if $\mathscr{S}$ does; hence we may assume without loss of generality that $\mathscr{S}$ is closed under finite direct sums. But then $I$ belongs to the quotient closure of $\mathscr{S} \otimes \bar{T}$ ([2], Theorem 1.1).

Let $C$ be a compact subset of $G$. For fixed $\delta>0$, choose $F$ and $\varepsilon$ as in Lemma 3. Let $r$ be the dimension of $H(T)$; and put $C^{\prime}=$ $(C \cup F) \cup(C \cup F)^{-1}$. 
By [2], Lemma 1.1, there is an $S$ in $\mathscr{S}$ and a unit vector $\zeta$ in $H(S \otimes \bar{T})$ such that

$$
\left\|(S \otimes \bar{T})_{x} \zeta-\zeta\right\|<\frac{\varepsilon}{2 r^{4}}
$$

for all $x$ in $C^{\prime}$. Fixing an orthonormal basis $\xi_{1}, \cdots, \xi_{r}$ of $H(T)$, let us write $\zeta=\sum_{i=1}^{r} \eta_{i} \otimes \xi_{i}\left(\eta_{i} \in H(S)\right)$, where

$$
1=\|\zeta\|^{2}=\sum_{i=1}^{r}\left\|\eta_{i}\right\|^{2}
$$

If the matrix of $T_{x}$ in the basis $\left\{\xi_{i}\right\}$ is $\left\{\tau_{i j}(x)\right\}$, we have $\bar{T}_{x} \xi_{i}=$ $\sum_{j=1}^{r} \overline{\tau_{j i}(x)} \xi_{j}$. So $(S \otimes \bar{T})_{x} \zeta=\sum_{j}\left(\sum_{i} \overline{\tau_{j i}(x)} S_{x} \eta_{i}\right) \otimes \xi_{j}$, whence

$$
\left\|(S \otimes \bar{T})_{x} \zeta-\zeta\right\|^{2}=\sum_{j}\left\|\left(\sum_{i} \overline{\tau_{j i}(x)} S_{x} \eta_{i}\right)-\eta_{j}\right\|^{2} .
$$

By (4) and (6),

$$
\left\|\left(\sum_{i} \overline{\tau_{j i}(x)} S_{x} \eta_{i}\right)-\eta_{j}\right\|<\frac{\varepsilon}{2 r^{4}}
$$

$\left(x \in C^{\prime}, j=1, \cdots, r\right)$. From (7) and the unitariness of $\tau(x)$,

$$
\begin{aligned}
\| S_{x} \eta_{k} & -\sum_{j} \tau_{j k}(x) \eta_{j} \| \\
& \leqq \sum_{j}\left|\tau_{j k}(x)\right|\left\|\left(\sum_{i} \overline{\tau_{j i}(x)} S_{x} \eta_{i}\right)-\eta_{j}\right\| \\
& <\frac{\varepsilon}{2 r^{3}} .
\end{aligned}
$$

Let $A$ be the linear map of $H(T)$ into $H(S)$ sending $\xi_{i}$ into $\eta_{i}(i=$ $1, \cdots, r)$. Then (8) gives

$$
\left\|S_{x} A-A T_{x}\right\|<\frac{\varepsilon}{2 r^{2}}
$$

From this and the symmetry of $C^{\prime}$,

$$
\left\|A^{*} S_{x}-T_{x} A^{*}\right\|<\frac{\varepsilon}{2 r^{2}}
$$

By (5), $\|A\|=\left\|A^{*}\right\| \leqq r$ and also

$$
\left\|A^{*} A\right\| \geqq \frac{1}{r}
$$

Hence, denoting $A^{*} A /\left\|A^{*} A\right\|$ by $B$, we obtain from (9) and (10) $\left\|B T_{x}-T_{x} B\right\|<\varepsilon\left(x \in C^{\prime}\right)$. Since $B$ is positive, $\|B\|=1$, and $F \subset C^{\prime}$, 
Lemma 3 asserts that $\|B-E\|<\delta$. From this, setting $\eta_{i}^{\prime}=\eta_{i} /\|A\|$, we get

$$
\left|\left(\eta_{i}^{\prime}, \eta_{j}^{\prime}\right)-\delta_{i j}\right|<\delta
$$

for all $i, j$. Let $\varphi(x)=\left(S_{x} \eta_{1}^{\prime}, \eta_{1}^{\prime}\right)(x \in G)$. By (8) and (11) $\| S_{x} \eta_{1}^{\prime}$ $\sum_{j} \tau_{j_{1}}(x) \eta_{j}^{\prime} \|<\varepsilon / 2 r^{2}$. Combining this with (12) we have for $x$ in $C$

$$
\begin{aligned}
& \left|\varphi(x)-\tau_{11}(x)\right| \leqq\left|\left(\left(S_{x} \eta_{1}^{\prime}-\sum_{j} \tau_{j_{1}}(x) \eta_{j}^{\prime}\right), \eta_{1}^{\prime}\right)\right| \\
& \quad+\left|\left(\sum_{j} \tau_{j_{1}}(x) \eta_{j}^{\prime}, \eta_{1}^{\prime}\right)-\tau_{11}(x)\right| \\
& \leqq \\
& \sum_{j}\left|\tau_{j_{1}}(x)\right|\left|\left(\eta_{j}^{\prime}, \eta_{1}^{\prime}\right)-\delta_{j 1}\right|+\frac{\varepsilon}{2 r^{2}}\left\|\eta_{1}^{\prime}\right\| \\
& \leqq \\
& \quad r \delta+\frac{\varepsilon}{2 r}
\end{aligned}
$$

which is as small as we wish. Thus we have an $S$ in $\mathscr{S}$ and a function. $\varphi$ of positive type associated with $S$ which differs from $\tau_{11}$ on $C$ by an arbitrarily small quantity. So $\mathscr{S}$ weakly contains $T$.

Combining Lemmas 2 and 4 we get:

THEOREM 4. Let $\mathscr{S}$ be a family of unitary representations of $G$ and $T$ a finite-dimensional irreducible unitary representation of $G$. Then $\mathscr{S}$ weakly contains $T$ if and only if $\mathscr{S} \otimes \bar{T}$ weakly contains $I$.

As a corollary we mention the following weak "Frobenius-like" proposition. As usual, $U^{S}$ denotes the representation of $G$ induced from the representation $S$ of a subgroup.

Corollary. Let $K$ be a closed subgroup of $G$, and $J$ and $I$ the identity representations of $K$ and $G$ respectively. We assume that $U^{J}$ weakly contains $I$. If $\mathscr{S} \subset \mathscr{T}(K), T$ is a finite-dimensional irreducible unitary representation of $G$, and $\mathscr{S}$ weakly contains some. irreducible component of $T \mid K$, then $\left\{U^{S} \mid S \in \mathscr{P}\right\}$ weakly contains $T$.

Proof. By Theorem $4 \mathscr{S} \otimes \bar{T} \mid K$ weakly contains $J$. Hence by [2], Theorem 4.2, $\left\{U^{s \bar{\nabla} \bar{T} \mid K} \mid S \in \mathscr{S}\right\}$ weakly contains $U^{J}$. By hypothesis. the latter weakly contains $I$; so $\left\{U^{S \diamond \bar{T}} \mathbb{S} \mid S \in \mathscr{S}\right\}$ weakly contains $I$. But by [2], Lemma 4.2, $U^{s \otimes T}, K \cong U^{S} \otimes \bar{T}$. Hence another application of Theorem 4 gives the required conclusion.

3. A counter-example. Let $G$ be the proper Euclidean group in three-dimensional real space $R^{3}$. We observe that the hull-kernel 
topology of $\hat{G}$ is $T_{1}$ (i.e. points are closed). Indeed, the results of [5] show that $T_{f}$ is completely continuous whenever $T \in \hat{G}$ and $f \in L_{1}(G)$. So, by [1], Lemma 1.11, $\hat{G}$ is $T_{1}$. Thus, if $S$ and $T$ are inequivalent elements of $\hat{G}$, neither weakly contains the other. We shall now construct two inequivalent elements $S$ and $T$ of $\hat{G}$ such that $S \otimes \bar{T}$ weakly contains $I$ (see the beginning of $\S 2$ ).

Let $N$ and $K$ be the translation and rotation subgroups of $G$ respectively; $\tau_{u}$ will denote translation by $u: \tau_{u}(v)=u+v\left(u, v \in R^{3}\right)$. Let $\chi$ be the fixed character of $N$ defined by $\chi\left(\tau_{u}\right)=e^{i u_{1}}$. The "stationary subgroup" for $\chi$ (consisting of those $\sigma$ in $G$ such that $\chi\left(\sigma \tau_{u} \sigma^{-1}\right)=\chi\left(\tau_{u}\right)$ for all $\left.u\right)$ is $Z=H N$, where $H=\{\rho \in K \mid \rho(1,0,0)=$ $(1,0,0)\}$. Thus, by [6], Theorem 14.1, to each character $\varphi$ of the Abelian group $H$ we get an irreducible representation $T^{\varphi}$ of $G$, namely, that induced from the character $\psi$ of $Z$, where

$$
\psi\left(\rho \tau_{u}\right)=\varphi(\rho) \chi\left(\tau_{u}\right)
$$

$\left(\rho \in H, u \in R^{3}\right)$.

Further, if $\varphi$ and $\varphi^{\prime}$ are distinct characters of $H, T^{\varphi}$ and $T^{\varphi^{\prime}}$ are inequivalent.

Now let $\varphi$ and $\varphi^{\prime}$ be distinct characters of $H$. Let $0<\theta<\pi / 2$ and let $\rho$ be the element of $K$ consisting of rotation through an angle $\theta$ about the third axis. We verify easily that $Z \cap \rho Z \rho^{-1}=N$. Hence by [3], Theorem 5.4 (the 'weak containment' version of Mackey's Kronecker Product Theorem), $T^{\varphi} \otimes\left(T^{\varphi^{\prime}}\right)^{-}$weakly contains the representation of $G$ induced from the character $\chi_{\theta}$ of $N$ given by $\chi_{\theta}\left(\tau_{u}\right)=$ $\chi\left(\tau_{\rho(u)}\right) \chi\left(\tau_{u}\right)$. (Here $\left(T^{\varphi^{\prime}}\right)^{-}$is the adjoint of $\left.T^{\varphi^{\prime}}\right)$. Since this is true whenever $0<\theta<\pi / 2$, we can use [2], Theorem 4.2 , to pass to the limit as $\theta \rightarrow 0$; we then conclude that $T^{\varphi} \otimes\left(T^{\varphi^{\prime}}\right)^{-}$weakly contains $U^{x_{0}}$, where $\chi_{0}$ is the identity character of $N$. But $U^{x_{0}}$ is obtained by lifting to $G$ the regular representation of the compact group $K$; hence it contains $I$ as a direct summand. Thus we conclude that $T^{\varphi} \otimes\left(T^{\varphi^{\prime}}\right)^{-}$ weakly contains $I$. This is the desired example, since we have already observed that $T^{\varphi}$ and $T^{\varphi^{\prime}}$ are inequivalent irreducible representations of $G$.

\section{BIBLIOGRAPHY}

1. J. M. G. Fell, The dual spaces of $C^{*}$-algebras, Trans. Amer. Math. Soc., 94 (1960), 365-403.

2. Weak containment and induced representations of groups, Can. J. Math., 14 (1962), 237-268.

3. - Weak containment and induced representations of groups, $I I$, to appear in Trans. Amer. Math. Soc.

4. R. Godement, Les fonctions de type positif et la théorie des groupes, Trans. Amer. Math. Soc., 63 (1948), 1-84.

5. — A theory of spherical functions, I, Trans. Amer. Math. Soc., 73 (1952), 496-556. 
6. G. W. Mackey, Induced representations of locally compact groups, I, Ann. Math., 55 (1952), 101-139.

7. Mimeographed Notes on Unitary Representations of Groups, University of Chicago, 1955.

8. O. Takenouchi, Sur une classe de fonctions continues de type positif sur un groupe localement compact, Math. J. Okayama Univ., 4 (1955), 143-173. 


\title{
PACIFIC JOURNAL OF MATHEMATICS
}

\author{
EDITORS
}

\author{
Ralph S. Phillips \\ Stanford University \\ Stanford, California \\ M. G. Arsove \\ University of Washington \\ Seattle 5 , Washington
}

\author{
J. DugunduI \\ University of Southern California \\ Los Angeles 7, California
}

Lowell J. Paige

University of California

Los Angeles 24, California

\section{ASSOCIATE EDITORS}

E. F. BECKENBACH

T. M. CHERRY
D. DERRY
M. OHTSUKA

H. L. ROYDEN

E. SPANIER
E. G. STRAUS

F. WOLF

\section{SUPPORTING INSTITUTIONS}

UNIVERSITY OF BRITISH COLUMBIA CALIFORNIA INSTITUTE OF TECHNOLOGY UNIVERSITY OF CALIFORNIA MONTANA STATE UNIVERSITY UNIVERSITY OF NEVADA NEW MEXICO STATE UNIVERSITY OREGON STATE UNIVERSITY UNIVERSITY OF OREGON OSAKA UNIVERSITY UNIVERSITY OF SOUTHERN CALIFORNIA
STANFORD UNIVERSITY

UNIVERSITY OF TOKYO

UNIVERSITY OF UTAH

WASHINGTON STATE UNIVERSITY

UNIVERSITY OF WASHINGTON

AMERICAN MATHEMATICAL SOCIETY CALIFORNIA RESEARCH CORPORATION SPACE TECHNOLOGY LABORATORIES NAVAL ORDNANCE TEST STATION

Mathematical papers intended for publication in the Pacific Journal of Mathematics should be typewritten (double spaced), and the author should keep a complete copy. Manuscripts may be sent to any one of the four editors. All other communications to the editors should be addressed to the managing editor, L. J. Paige at the University of California, Los Angeles 24, California.

50 reprints per author of each article are furnished free of charge; additional copies may be obtained at cost in multiples of 50 .

The Pacific Journal of Mathematics is published quarterly, in March, June, September, and December. Effective with Volume 13 the price per volume (4 numbers) is $\$ 18.00$; single issues, $\$ 5.00$. Special price for current issues to individual faculty members of supporting institutions and to individual members of the American Mathematical Society: $\$ 8.00$ per volume; single issues \$2.50. Back numbers are available.

Subscriptions, orders for back numbers, and changes of address should be sent to Pacific Journal of Mathematics, 103 Highland Boulevard, Berkeley 8, California.

Printed at Kokusai Bunken Insatsusha (International Academic Printing Co., Ltd.), No. 6, 2-chome, Fujimi-cho, Chiyoda-ku, Tokyo, Japan.

PUBLISHED BY PACIFIC JOURNAL OF MATHEMATICS, A NON-PROFIT CORPORATION

The Supporting Institutions listed above contribute to the cost of publication of this Journal, but they are not owners or publishers and have no responsibility for its content or policies. 


\section{Pacific Journal of Mathematics}

\section{Vol. 13, No. 2 \\ April, 1963}

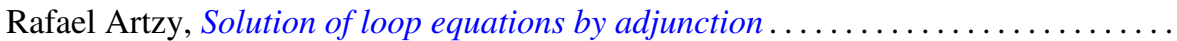

Earl Robert Berkson, A characterization of scalar type operators on reflexive

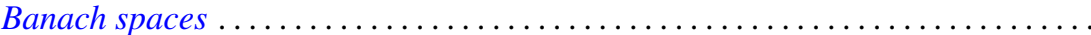

Mario Borelli, Divisorial varieties

365

Raj Chandra Bose, Strongly regular graphs, partial geometries and partially

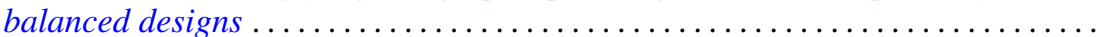

389

R. H. Bruck, Finite nets. II. Uniqueness and imbedding ............... 421

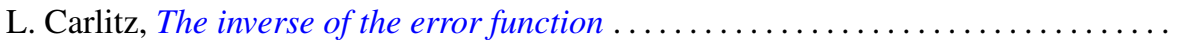

Robert Wayne Carroll, Some degenerate Cauchy problems with operator

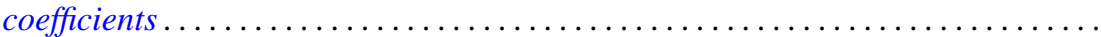

Michael P. Drazin and Emilie Virginia Haynsworth, A theorem on matrices of 0 's

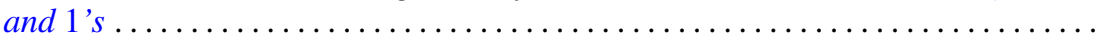

Lawrence Carl Eggan and Eugene A. Maier, On complex approximation .......... James Michael Gardner Fell, Weak containment and Kronecker products of group

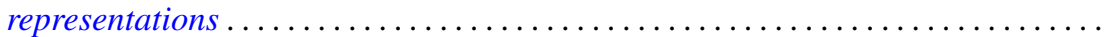

Paul Chase Fife, Schauder estimates under incomplete Hölder continuity

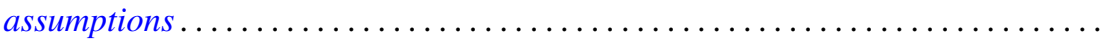

Shaul Foguel, Powers of a contraction in Hilbert space ...................

Neal Eugene Foland, The structure of the orbits and their limit sets in continuous

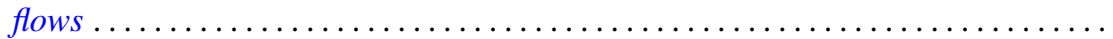

Frank John Forelli, Jr., Analytic measures . . . . . . . . . . . . . . . . . . . . . 563

Robert William Gilmer, Jr., On a classical theorem of Noether in ideal theory ....... P. R. Halmos and Jack E. McLaughlin, Partial isometries .

Albert Emerson Hurd, Maximum modulus algebras and local approximation in

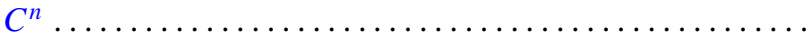

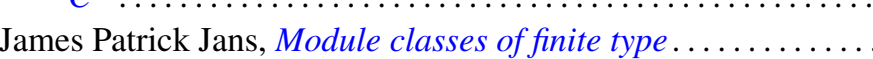

Betty Kvarda, On densities of sets of lattice points ...

H. Larcher, A geometric characterization for a class of discontinuous groups of linear fractional transformations .

John W. Moon and Leo Moser, Simple paths on polyhedra .

T. S. Motzkin and Ernst Gabor Straus, Representation of a point of a set as sum of

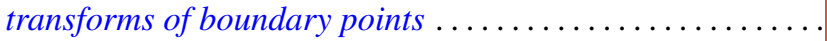

Rajakularaman Ponnuswami Pakshirajan, An analogue of Kolmogorov's three-series theorem for abstract random variables ...............

Robert Ralph Phelps, Čebyšev subspaces of finite codimension in $C(X)$...

James Dolan Reid, On subgroups of an Abelian group maximal disjoint from a given subgroup ...

William T. Reid, Riccati matrix differential equations and non-oscillation criteria for associated linear differential systems .................

Georg Johann Rieger, Some theorems on prime ideals in algebraic number fields ...

Gene Fuerst Rose and Joseph Silbert Ullian, Approximations of functions on the

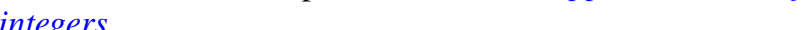

F. J. Sansone, Combinatorial functions and regressive isols . . . . . . . . . . 703

Leo Sario, On locally meromorphic functions with single-valued moduli . . . . . . . 709

Takayuki Tamura, Semigroups and their subsemigroup lattices.

Pui-kei Wong, Existence and asymptotic behavior of proper solutions of a class of second-order nonlinear differential equations . . ........... 